\title{
PD-L1 expression as a prognostic marker in patients with advanced biliary tract cancer
}

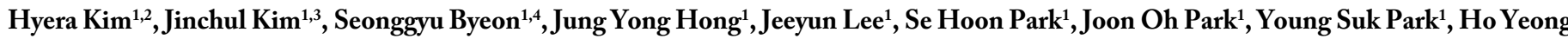 \\ Lim $^{1}$, Won Ki Kang ${ }^{1}$ and Seung Tae Kim ${ }^{1 *}$ \\ ${ }^{1}$ Division of Hematology-Oncology, Department of Medicine, Samsung Medical Center, Sungkyunkwan University School of Medicine, Seoul, South Korea \\ ${ }^{2}$ Division of Hematology-Oncology, Department of Internal Medicine, Keimyung University Dongsan Hospital, Daegu, South Korea \\ ${ }^{3}$ Department of Hematology-Oncology, Inha University College of Medicine and Hospital, Incheon, South Korea \\ ${ }^{4}$ Department of Internal Medicine, Chungbuk National University Hospital, Chungbuk National University College of Medicine, Cheongju, South Korea
}

\begin{abstract}
Background: Biliary tract cancer (BTC) is associated with poor prognosis because of its aggressive and heterogeneous nature. Programmed death ligand 1 (PD-L1) has been considered as a novel biomarker for prognosis and response of immune checkpoint inhibitors in various tumors. However, there are limited data reporting on the role of PD-L1 in advanced BTC patients.

Methods: We analyzed 186 patients with advanced BTC who received palliative gemcitabine and platinum between May 2010 and December 2019. All patients were evaluated for PD-L1 expression by combined positive score positivity.

Results: In all 186 patients, the primary tumor location was intrahepatic cholangiocarcinoma (IH-CCC) in 72 patients (38.7\%), extrahepatic cholangiocarcinoma (EH-CCC) in 90 (48.4\%), and gallbladder (GB) cancer in 24 (12.9\%). Among the 186 patients, 53 (28.5\%) had PD-L1 positivity, and 133 were PD-L1 negative. The median overall survival (OS) of patients with PD-L1 positivity or negativity was 12.1 and 15.4 months, respectively. The median progression-free survival (PFS) in patients with PD-L1 positivity or negativity was 5.7 and 7.1 months, respectively. The OS and PFS were not statistically different between groups. In subgroup analysis, EH-CCC patients with PD-L1 negativity had more favorable OS (17.2 vs. 11.6 months, $\mathrm{p}=0.002)$ and PFS $(7.8$ vs. 5.4 months, $\mathrm{p}=0.005)$ than those that were PD-L1 positive. However, this finding was not reproduced in patients with IH-CCC or GB cancer.
\end{abstract}

Conclusion: This study demonstrated that PD-L1 expression might be a novel prognostic biomarker in patients with EH-CCC but not for patients with IH-CCC or GB cancer.

\section{Introduction}

Biliary tract cancer (BTC), including intra- (IH) and extra-hepatic (EH) cholangiocarcinoma (CCC) and gallbladder (GB) cancer, has poor prognosis because of the aggressive and heterogeneous nature, despite curative resection [1-3]. Resectable diseases are reported in less than $30 \%$ of BTC cases at diagnosis, and the 5 -year survival rate can be as low as $20 \%$ to $40 \%$ due to frequent recurrence and lack of effective treatment $[4,5]$. Although surgical techniques and postoperative management have improved, BTC prognosis remains poor. Therefore, effective palliative therapy and appropriate biomarkers are needed for advanced BTC patients $[3,6]$.

Recently, immune checkpoint inhibitors (CPIs) that target programmed cell death protein 1 (PD-1) or programmed cell death protein ligand 1 (PD-L1) have shown promising outcomes and have emerged as standard treatments for various types of cancer [7-9]. The PD-1 on the surface of activated T cells binds to PD-L1, expressed on the surface of tumor cells, and this binding induces $\mathrm{T}$ cell apoptosis and suppresses autoimmunity to tumor cells, which allows the tumor cells to escape the immune system $[10,11]$. These immune-inhibitory signals can be blocked by immune checkpoint inhibitors, which also influence the tumor microenvironment [12]. Therefore, PD-L1 expression is considered a predictive marker for tumor response of immune CPIs [13].
PD-L1 expression has been reported to be associated with poor prognosis in malignancies. In the tumor microenvironment, various immune cells produce an immunosuppressive environment that accelerates cancer progression, and PD-L1 expression affects immune cell infiltration and immune evasion [14,15]. However, there are limited numbers of studies that have shown a correlation between high PD-L1 expression and low OS in patients with BTC $[16,17]$. However, these reports have been based on resectable diseases, and there are limited data on the role of PD-L1 in advanced BTC patients.

Therefore, we evaluated the prognostic value of PD-L1 expression in patients with advanced BTC and assessed subpopulations based on the clinical significance of PD-L1 expression.

${ }^{\star}$ Correspondence to: Seung Tae Kim, M.D., Ph.D., Division of HematologyOncology, Department of Medicine, Samsung Medical Center, Sungkyunkwan University School of Medicine, 81 Irwon-ro, Gangnam-gu, Seoul 06351, South Korea, Tel: +82-2-3410-0297; Fax: +82-2-3410-1754; E-mail: seungtae1.kim@ samsung.com

Key words: biliary tract cancer, extrahepatic cholangiocarcinoma, PD-L1 expression, Prognosis

Received: September 28, 2020; Accepted: October 05, 2020; Published: October 08, 2020 


\section{Methods and Patients}

\section{Patients}

We collected 186 patients with advanced BTC treated at Samsung Medical Center who received palliative gemcitabine and platinum between May 2010 and December 2019. Patients had histologically or cytologically confirmed BTC, which was defined as tumors of the IH- or EH-CCC or GB cancer. Pathologic diagnoses of BTC were based on the World Health Organization (WHO) classification. Ampulla of Vater cancer and duodenal cancer were excluded. Medical records were reviewed for age, sex, date of diagnosis, primary tumor location, sites and numbers of metastases, history of treatment including surgery and palliative chemotherapy, progression, and death. The plans and schedules of palliative chemotherapy depended on the clinician. Treatment response was assessed with CT scans, based on the Response Evaluation Criteria in Solid Tumors (RECIST) version 1.1. Times from the date of the first palliative chemotherapy to the date of death [overall survival (OS)] or that of disease progression or death [progression-free survival (PFS)] were calculated for each patient. This study was approved by the Institutional Review Board of Samsung Medical Center.

\section{PD-L1 Immunohistochemistry}

All patients were evaluated for PD-L1 expression by combined positive score (CPS) positivity. Tissue sections were freshly cut to 4 um, mounted on Fisher Superfrost Plus Microscope Slides (Thermo Fisher Scientific, Waltham, MA), and dried at $60^{\circ} \mathrm{C}$ for 1 hour. IHC staining was carried out with the Dako Autostainer Link 48 system (Agilent Technologies, Santa Clara, CA) using a Dako PD-L1 IHC 22C3 pharmDx kit (Agilent Technologies) with an EnVision FLEX visualization system. The slides were counterstained with hematoxylin according to the manufacturer's instructions. PD-L1 protein expression was determined using the Combined Positive Score (CPS), which is the number of PDL1-stained cells (tumor cells, lymphocytes, macrophages) divided by the total number of viable tumor cells, multiplied by 100 . A specimen was considered to have PD-L1 expression if CPS $\geq 1$.

\section{Statistical analysis}

Differences between the two groups were assessed using Student's $\mathrm{t}$ test or the Mann-Whitney $\mathrm{U}$ test for continuous variables and the Chi-square test or Fisher's exact test for categorical variables. OS and PFS were estimated by the Kaplan-Meier method and were compared using the log-rank test. A Cox proportional hazard regression model was performed to identify the prognostic prediction of PD-L1 expression in each subgroup. The results were presented as hazard ratio (HR) and $95 \%$ confidence interval (CI). P values less than 0.05 were considered significant. All statistical analyses were computed using IBM SPSS Statistics for Windows, version 25.0 (IBM Corp., Armonk, NY, USA).

\section{Results}

\section{Patient characteristics}

A total of 186 patients was analyzed in this study, and the clinicpathologic characteristics of patients are presented in Table 1. The median age was 62 years (range 38-82 years), and 115 (61.8\%) patients were male. The primary tumor location was IH-CCC in 72 patients (38.7\%), EH-CCA in 90 (48.4\%), and GB cancer in 24 (12.9\%). Sixtyone $(32.8 \%)$ patients had more than one metastatic lesion, and the most common metastatic site was the liver $(n=85,45.7 \%)$ All patients received gemcitabine and platinum as first-line therapy, and response to treatment was CR in 1 (0.5\%), PR in 31 (16.7\%), and SD in $102(54.8 \%)$ patients. There were no differences in these clinico-pathologic features based on primary tumor location.

\section{PD-L1 expression}

Among the 186 patients, 53 (28.5\%) had PD-L1 CPS positivity based on tumor samples. PD-L1 CPS positivity was similarly distributed among the primary tumor locations (IH-CCC, EH-CCC, and GB cancer). There were no statistically significant differences in clinicopathologic features between patients based on PD-L1 CPS positivity. Group comparisons are described in Table 2.

Table 1. Characteristics of patients with advanced BTC according to primary tumor location

\begin{tabular}{|c|c|c|c|c|}
\hline \multicolumn{2}{|l|}{ Characteristics } & \multirow{2}{*}{$\begin{array}{l}\text { Intra-hepatic CCC }(\mathbf{n}=\mathbf{7 2}) \\
61.0(38-76)\end{array}$} & \multirow{2}{*}{$\begin{array}{l}\text { Extra-hepatic CCC }(\mathbf{n}=90) \\
63.5(46-82)\end{array}$} & \multirow{2}{*}{$\begin{array}{l}\text { GB cancer }(\mathbf{n}=\mathbf{2 4}) \\
60.0(44-74)\end{array}$} \\
\hline \multirow{3}{*}{ Age (years) } & Median (range) & & & \\
\hline & $>60$ & $38(52.8)$ & $55(61.1)$ & $11(45.8)$ \\
\hline & $\leq 60$ & $34(47.2)$ & $35(38.9)$ & $13(54.2)$ \\
\hline \multirow[t]{2}{*}{ Sex } & Male & $43(59.7)$ & $62(68.9)$ & $10(41.7)$ \\
\hline & Female & $29(40.3)$ & $28(31.1)$ & $14(58.3)$ \\
\hline \multirow{2}{*}{ Disease status } & Recurrence & $51(70.8)$ & $84(93.3)$ & $23(95.8)$ \\
\hline & Metastasis & $21(29.2)$ & $6(6.7)$ & $1(4.2)$ \\
\hline \multirow{2}{*}{ Liver metastasis } & Yes & $35(48.6)$ & $38(42.2)$ & $12(50.0)$ \\
\hline & No & $37(51.4)$ & $52(57.8)$ & $12(50.0)$ \\
\hline \multirow{2}{*}{ No. of metastatic sites } & 1 & $40(55.6)$ & $65(72.2)$ & $20(83.3)$ \\
\hline & $>1$ & $32(44.4)$ & $25(27.8)$ & $4(16.7)$ \\
\hline \multicolumn{5}{|c|}{ First-line palliative chemotherapy } \\
\hline \multirow{5}{*}{ Best response } & $\mathrm{CR}$ & $1(1.4)$ & $0(0.0)$ & $0(0.0)$ \\
\hline & PR & $13(18.1)$ & $15(16.7)$ & $3(12.5)$ \\
\hline & SD & $37(51.4)$ & $56(62.2)$ & $9(37.5)$ \\
\hline & PD & $11(15.3)$ & $11(12.2)$ & $9(37.5)$ \\
\hline & NA & $10(13.9)$ & $8(8.9)$ & $3(12.5)$ \\
\hline \multirow{2}{*}{$\begin{array}{l}\text { No. of lines of palliative } \\
\text { chemotherapy }\end{array}$} & 1 & $45(62.5)$ & $62(68.9)$ & $15(62.5)$ \\
\hline & $>1$ & $27(37.5)$ & $28(31.1)$ & $9(37.5)$ \\
\hline
\end{tabular}

$C C C$ cholangiocarcinoma, $G B$ gallbladder, No. number, $C R$ complete response, $P R$ partial response, $S D$ stable disease, $P D$ progressive disease, $N A$ not available. 


\section{Survival outcome and PD-L1 CPS positivity in patients with advanced BTC}

In 186 patients with advanced BTC, the median OS and PFS after first-line gemcitabine and platinum were 14.1 months (CI 11.94116.259) and 6.8 months (CI 5.983-7.683), respectively. The results indicated no significant difference for OS and PFS after first-line therapy between patients with PD-L1 CPS positivity or negativity (OS; 12.1 months vs. 15.4 months, $\mathrm{p}=0.086$ and PFS; 5.7 months vs. 7.1 months, $\mathrm{p}=0.089$ ) (Figure 1A and 1B). Next, we analyzed the influence of PD-L1 on survival based on primary tumor location (IH-CCC, HHCCC, and GB cancer). In EH-CCC, patients with PD-L1 CPS negativity showed more favorable OS (17.2 months vs. 11.6 months, $\mathrm{p}=0.002)$ and PFS (7.8 months vs. 5.4 months, $\mathrm{p}=0.005)$ compared to those with PD-L1 CPS positivity (Figure 2A and 2B). However, this finding was not reproduced in patients with IH CCC (OS; 15.8 months vs. 14.4 months, $\mathrm{p}=0.953$ and PFS; 7.1 months vs. 5.7 months, $\mathrm{p}=0.586$, Figure $2 \mathrm{C}$ and $2 \mathrm{D}$ ) or GB cancer (OS; 10.7 months vs. 10.9 months, $\mathrm{p}=0.539$ and PFS; 3.6 months vs. 6.3 months, $\mathrm{p}=0.409$, Figure $2 \mathrm{E}$ and $2 \mathrm{~F}$ ).

Univariate analysis of the association between PD-L1 expression and OS in patients with advanced BTC indicated that PD-L1 CPS positivity has a prognostic role in subpopulations older than 60 years (HR 1.743, CI 1.001-3.034, p = 0.050), those with EH-CCA (HR 2.449, CI 1.355-4.426, $\mathrm{p}=0.003$ ), and those with liver metastasis (HR 2.511, CI 1.362-4.626, $\mathrm{p}=0.003$ ) (Figure 3 ).

Table 2. Characteristics of patients with advanced BTC according to PD-L1 expression

\begin{tabular}{|c|c|c|c|c|c|}
\hline \multicolumn{2}{|l|}{ Characteristics } & \multirow[t]{2}{*}{$\begin{array}{l}\text { Total } \\
(\mathbf{n}=\mathbf{1 8 6})\end{array}$} & \multicolumn{3}{|c|}{ PD-L1 expression } \\
\hline & & & $\begin{array}{l}\text { Positive } \\
(\mathrm{n}=53)\end{array}$ & $\begin{array}{l}\text { Negative } \\
(\mathrm{n}=133)\end{array}$ & $P$ \\
\hline \multirow{3}{*}{ Age (years) } & Median (range) & $62(38-82)$ & $62(45-82)$ & $62(38-77)$ & 0.696 \\
\hline & $>60$ & $104(55.9)$ & $29(54.7)$ & $75(56.4)$ & 0.836 \\
\hline & $\leq 60$ & $82(44.1)$ & $24(45.3)$ & $58(43.6)$ & \\
\hline \multirow[t]{2}{*}{ Sex } & Male & $115(61.8)$ & $29(54.7)$ & $86(64.7)$ & 0.208 \\
\hline & Female & $71(38.2)$ & $24(45.3)$ & $47(35.3)$ & \\
\hline \multirow{3}{*}{ Tumor location } & Intrahepatic CCC & $72(38.7)$ & $18(34.0)$ & $54(40.6)$ & 0.691 \\
\hline & Extrahepatic CCC & $90(48.4)$ & $28(52.8)$ & $62(46.6)$ & \\
\hline & GB cancer & $24(12.9)$ & $7(13.2)$ & $17(12.8)$ & \\
\hline \multirow{2}{*}{ Disease status } & Recurrence & $158(84.9)$ & $46(86.8)$ & $112(84.2)$ & 0.657 \\
\hline & Metastasis & $28(15.1)$ & $7(13.2)$ & $21(15.8)$ & \\
\hline \multirow{2}{*}{ Liver metastasis } & Yes & $85(45.7)$ & $21(39.6)$ & $64(48.1)$ & 0.294 \\
\hline & No & $101(54.3)$ & $32(60.4)$ & $69(51.9)$ & \\
\hline \multirow{2}{*}{ No. of metastatic sites } & 1 & $125(67.2)$ & $36(67.9)$ & $89(66.9)$ & 0.895 \\
\hline & $>1$ & $61(32.8)$ & $17(32.1)$ & $44(33.1)$ & \\
\hline \multicolumn{6}{|c|}{ First-line palliative chemotherapy } \\
\hline \multirow{5}{*}{ Best response } & CR & $1(0.5)$ & $1(1.9)$ & $0(0.0)$ & 0.262 \\
\hline & PR & $31(16.7)$ & $7(13.2)$ & $24(18.0)$ & \\
\hline & SD & $102(54.8)$ & $29(54.7)$ & $73(54.9)$ & \\
\hline & PD & $31(16.7)$ & $12(22.6)$ & $19(14.3)$ & \\
\hline & NA & $21(11.3)$ & $4(7.5)$ & $17(12.8)$ & \\
\hline
\end{tabular}

$P D-L 1$ programmed cell death protein ligand 1, $C C C$ cholangiocarcinoma, $G B$ gallbladder, $N o$. number, $C R$ complete response, $P R$ partial response, $S D$ stable disease, $P D$ progressive disease, $N A$ not available.
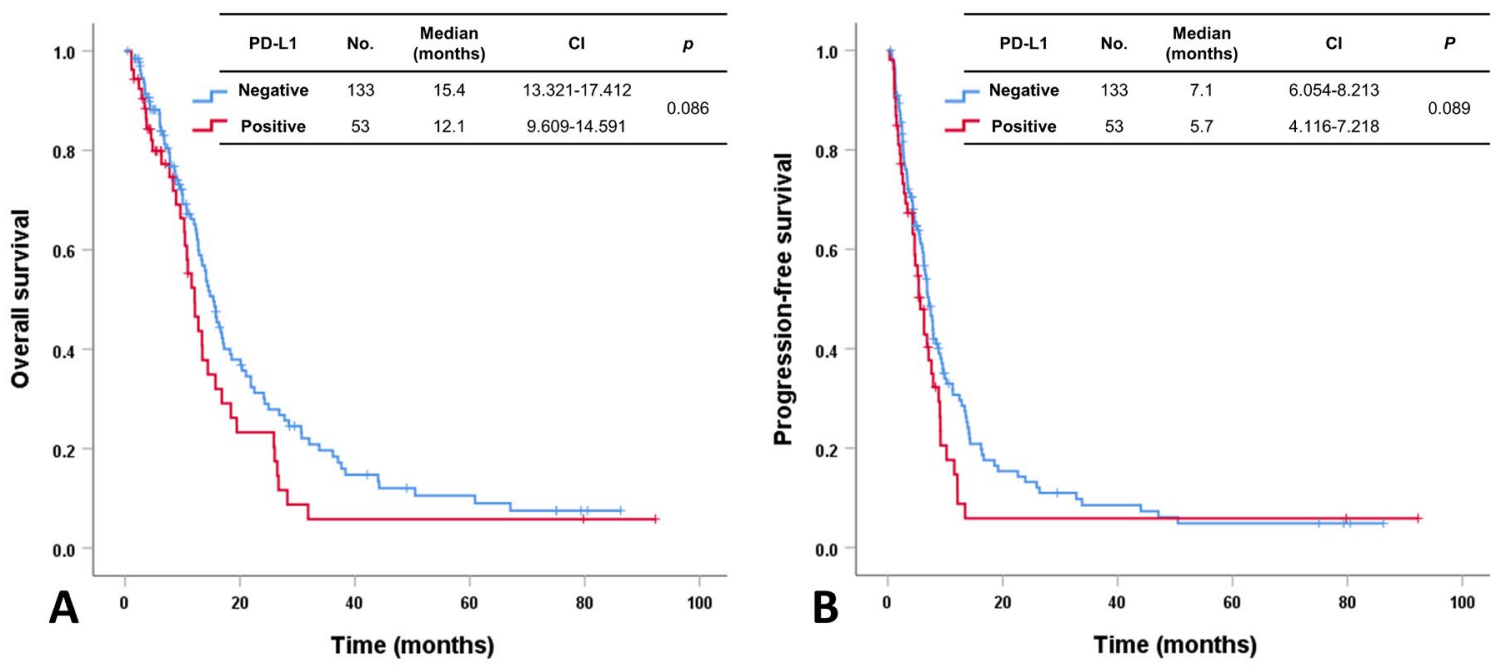

Figure 1. Overall survival and progression-free survival after first-line chemotherapy in patients with advanced BTC according to PD-L1 expression 

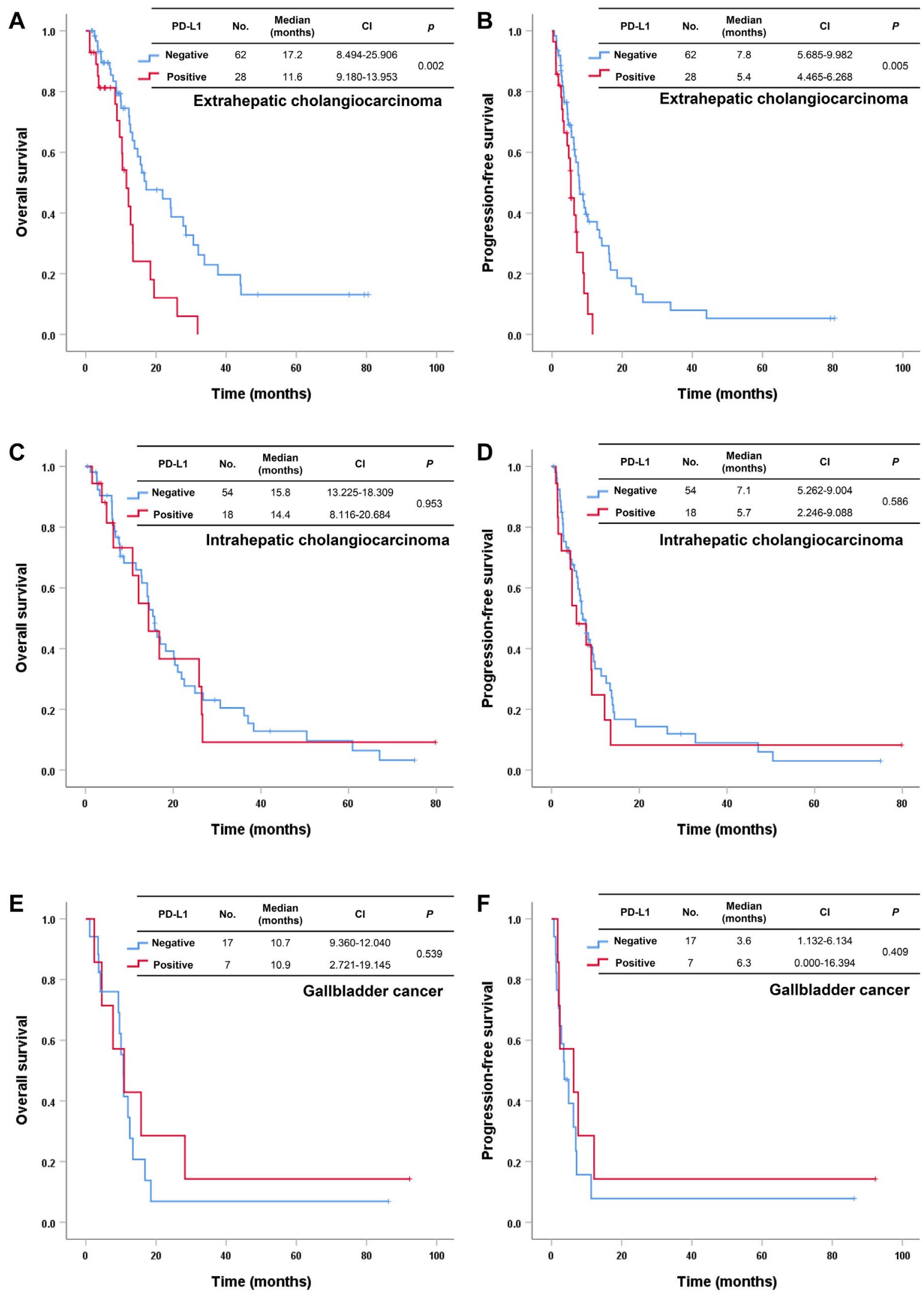

Figure 2. Overall survival and progression-free survival after first-line chemotherapy according to PD-L1 expression in patients with extrahepatic cholangiocarcinoma (A, B), intrahepatic cholangiocarcinoma $(\mathrm{C}, \mathrm{D})$, and gallbladder cancer $(\mathrm{E}, \mathrm{F})$ 


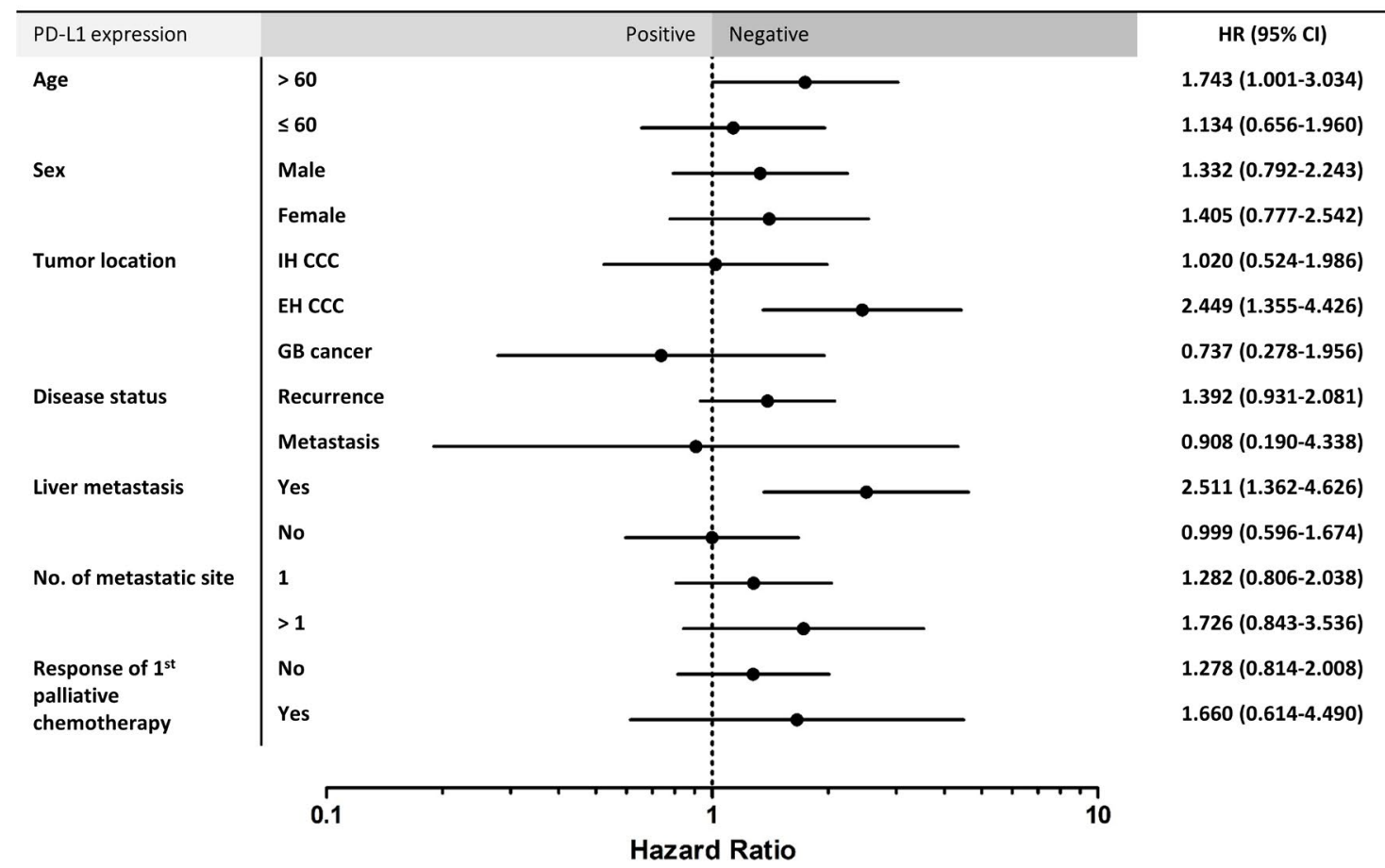

Figure 3. Subgroup analyses of the association between PD-L1 expression and overall survival in advanced BTC (N=186). PD-L1 programmed cell death protein ligand 1, HR hazard ratio, $C I$ confidence interval, $I H-C C C$ intrahepatic cholangiocarcinoma, EH-CCC extrahepatic cholangiocaricnoma, GB gallbladder, No., number

\section{Discussion}

This study investigated the impact of PD-L1 as a prognostic marker on patients with advanced BTC who received first-line gemcitabine and platinum. We evaluated the PD-L1 CPS in tumor samples of 186 advanced BTC cases, showing positivity in 53 (28.5\%). There was no statistically significant difference in OS or PFS after first-line therapy between patients with PD-L1 CPS positivity and negativity (OS; 12.1 months vs. 15.4 months, $\mathrm{p}=0.086$ and PFS; 5.7 months vs. 7.1 months, $\mathrm{p}=0.089$ ). Interestingly, in subgroup analysis, EH-CCC patients with PD-L1 negativity had more favorable OS and PFS than those with PDL1 positivity. However, this finding was not reproduced in patients with IH-CCC or GB cancer. These results suggest that PD-L1 has an important role as a biomarker in EH-CCC among BTC entities that consist of heterogeneous diseases, and that a treatment strategy targeted toward PD-L1 such as CPIs could be more beneficial for patients with advanced EH-CCC.

To date, the relationship between PD-L1 and BTC prognosis is not well understood. A meta-analysis of 1,066 patients from 11 studies reported that PD-L1 expression did not seem to be correlated with OS (HR 1.62, p = 0.063) [18]. However, several studies have advocated PDL1 expression is associated with poor prognosis in BTC. Kitano, et al. [17] evaluated 177 patients with CCC and found that PD-L1 expression in both cancer and stroma cells was a poor prognostic marker of OS (HR 2.20, $\mathrm{p}=0.002$ ). Lu, et al. [19] investigated the expression of PD1/ PD-L1 in 320 IH-CCC patients with heterogeneous characteristics and determined that high expression was a negative prognostic marker of OS and recurrence-free survival (RFS) for HBV-infected IH-CCC (OS, $\mathrm{p}=0.026 ; \mathrm{RFS}, \mathrm{p}=0.011$ ). In this study, we observed that PD-L1 was not a significant prognostic factor of OS and PFS for first-line gemcitabine and platinum in advanced BTC. However, in the EH-CCC subgroup, PDL1 was a statistically significant biomarker for OS and PFS. This finding should be investigated and validated in prospective BTC cohorts.
Chronic viral hepatitis and hepatolithiasis are major risk factors for BTC [20]. These factors produce repetitive inflammation and immune imbalance, resulting in carcinogenesis [21,22]. Immune CPIs may be effective because of high immunity and mutation burden through the mechanism mentioned. The earliest study of CPIs in BTC reported that deficiency-mismatch repair (dMMR) predicted the efficacy of pembrolizumab in solid tumor cancer patients, including only four BTC cases [23]. Recently, a phase II study, KEYNOTE-158, showed that pembrolizumab had a $5.8 \%$ overall response rate, although the median duration of response was not reached within 7.4 months of median OS in advanced BTCs, regardless of PD-L1 expression [24]. Results of clinical trials on efficacy of immune CPIs in advanced BTC patients have been disappointing. There is a limited amount of data on the correlation between PD-L1 expression and efficacy of CPIs. Further research is needed to appropriately assess CPIs and applications for advanced BTC.

There were several limitations to this study. First, it was retrospective and had heterogeneous patient cohorts that might influence findings due to selection bias. Second, the difficulty of obtaining a biopsy in BTC could affect the results of PD-L1 expression. Biopsy samples that were too small for analysis do not reflect intra- and/or inter-tumoral heterogeneity of PD-L1 expression. Third, the relationship between PD-L1 positivity and PD-L1 expression has not yet been defined. Finally, this study contained a small sample size. Nevertheless, this study evaluated the prognostic value of PD-L1 expression in patients with advanced BTC and worked to define subpopulations based on clinical significance of PD-L1 expression in advanced BTC patients.

In conclusion, this study demonstrated that PD-L1 expression might be a novel prognostic biomarker in patients with EH-CCC but not those with IH-CCC or GB cancer. Therefore, a treatment strategy targeted toward PD-L1 such as CPIs could be more beneficial for patients with advanced EH-CCC. 


\section{Funding}

No external funding was used in the preparation of this article.

\section{Conflict of interest}

The authors declare that they have no conflict of interest.

\section{Ethical approval}

This study was approved by the Institutional Review Board of Samsung Medical Center.

\section{References}

1. Anderson CD, Pinson CW, Berlin J, Chari RS (2004) Diagnosis and treatment of cholangiocarcinoma. Oncologist $9: 43-57$. [Crossref]

2. Nagino M, Ebata T, Yokoyama Y, Igami T, Sugawara G, et al. (2013) Evolution of surgical treatment for perihilar cholangiocarcinoma: a single-center 34-year review of 574 consecutive resections. Ann Surg 258: 129-140. [Crossref]

3. Takahashi Y, Ebata T, Yokoyama Y, Igami T, Sugawara G, et al. (2015) Surgery for Recurrent Biliary Tract Cancer: A Single-center Experience With 74 Consecutive Resections. Ann Surg 262: 121-129. [Crossref]

4. van der Gaag NA, Kloek JJ, de Bakker JK, Musters B, Geskus RB, et al. (2012) Survival analysis and prognostic nomogram for patients undergoing resection of extrahepatic cholangiocarcinoma. Ann Oncol 23: 2642-2649. [Crossref]

5. DeOliveira ML, Cunningham SC, Cameron JL, Kamangar F, Winter JM, et al. (2007) Cholangiocarcinoma: thirty-one-year experience with 564 patients at a single institution. Ann Surg 245: 755-762. [Crossref]

6. Murakami Y, Uemura K, Sudo T, Hashimoto Y, Nakashima A, et al. (2011) Prognostic factors after surgical resection for intrahepatic, hilar, and distal cholangiocarcinoma. Ann Surg Oncol 18: 651-658. [Crossref]

7. Garon EB, Rizvi NA, Hui R, Leighl N, Balmanoukian AS, et al. (2015) Pembrolizumab for the treatment of non-small-cell lung cancer. $N$ Engl J Med 372: 2018-2028. [Crossref]

8. Wolchok JD, Chiarion-Sileni V, Gonzalez R, Rutkowski P, Grob JJ, et al. (2017) Overall Survival with Combined Nivolumab and Ipilimumab in Advanced Melanoma. N Engl J Med 377: 1345-1356.

9. Overman MJ, Lonardi S, Wong KYM, Lenz HJ, Gelsomino F, et al. (2018) Durable Clinical Benefit With Nivolumab Plus Ipilimumab in DNA Mismatch Repair-Deficient/ Microsatellite Instability-High Metastatic Colorectal Cancer. J Clin Oncol 36: $773-$ 779. [Crossref]
10. Wei SC, Levine JH, Cogdill AP, Zhao Y, Anang NAS, et al. (2017) Distinct Cellular Mechanisms Underlie Anti-CTLA-4 and Anti-PD-1 Checkpoint Blockade. Cell 170: 1120-1133. [Crossref]

11. Topalian SL, Taube JM, Anders RA, Pardoll DM (2016) Mechanism-driven biomarkers to guide immune checkpoint blockade in cancer therapy. Nat Rev Cancer 16: 275-287. [Crossref]

12. Hegde PS, Karanikas V, Evers S (2016) The Where, the When, and the How of Immune Monitoring for Cancer Immunotherapies in the Era of Checkpoint Inhibition. Clin Cancer Res 22: 1865-1874. [Crossref]

13. Havel JJ, Chowell D, Chan TA (2019) The evolving landscape of biomarkers for checkpoint inhibitor immunotherapy. Nat Rev Cancer 19: 133-150. [Crossref]

14. Qian BZ, Pollard JW (2010) Macrophage diversity enhances tumor progression and metastasis. Cell 141: 39-51. [Crossref]

15. Boussiotis VA (2016) Molecular and Biochemical Aspects of the PD-1 Checkpoint Pathway. N Engl J Med 375: 1767-1778. [Crossref]

16. Gani F, Nagarajan N, Kim Y, Zhu Q, Luan L, et al. (2016) Program Death 1 Immune Checkpoint and Tumor Microenvironment: Implications for Patients With Intrahepatic Cholangiocarcinoma. Ann Surg Oncol 23: 2610-2617. [Crossref]

17. Kitano Y, Yamashita YI, Nakao Y, Itoyama R, Yusa T, et al. (2020) Clinical Significance of PD-L1 Expression in Both Cancer and Stroma Cells of Cholangiocarcinoma Patients. Ann Surg Oncol 27: 599-607. [Crossref]

18. Xu G, Sun L, Li Y, Xie F, Zhou X, et al. (2019) The Clinicopathological and Prognostic Value of PD-L1 Expression in Cholangiocarcinoma: A Meta-Analysis. Front Oncol 9: 897. [Crossref]

19. Lu JC, Zeng HY, Sun QM, Meng QN, Huang XY, et al. (2019) Distinct PD-L1/PD1 Profiles and Clinical Implications in Intrahepatic Cholangiocarcinoma Patients with Different Risk Factors. Theranostics 9: 4678-4687. [Crossref]

20. Tyson GL, El-Serag HB (2011) Risk factors for cholangiocarcinoma. Hepatology 54 173-184. [Crossref]

21. Tang LSY, Covert E, Wilson E, Kottilil S (2018) Chronic Hepatitis B Infection: A Review. JAMA 319: 1802-1813. [Crossref]

22. Labib PL, Goodchild G, Pereira SP (2019) Molecular Pathogenesis of Cholangiocarcinoma. BMC Cancer 19: 185. [Crossref]

23. Asaoka Y, Ijichi H, Koike K (2015) PD-1 Blockade in Tumors with Mismatch-Repair Deficiency. N Engl J Med 373 (20):1979. [Crossref]

24. Bang YJ, Ueno M, Malka D, Chung HC, Nagrial A, et al. (2019) Pembrolizumab (pembro) for advanced biliary adenocarcinoma: Results from the KEYNOTE-028 (KN028) and KEYNOTE-158 (KN158) basket studies. J Clin Oncol 37: 4079.

Copyright: (C2020 Kim H. This is an open-access article distributed under the terms of the Creative Commons Attribution License, which permits unrestricted use, distribution, and reproduction in any medium, provided the original author and source are credited. 